\title{
Impact of Heterogeneity on the Deployment of Sensor
}

\section{Networks}

\author{
Rabie A. Ramadan \\ Department of \\ Systems and Computer \\ Engineering \\ Al-Azhar University, \\ Cairo, Egypt. \\ 214-681-8049 \\ raramada@engr.smu.edu
}

\author{
Khaled Abdelghany \\ Department of \\ Environmental and Civil \\ Engineering \\ SMU, Dallas, TX 75275- \\ 0335, USA. \\ 214-768-3278 \\ khaled@engr.smu.edu
}

\author{
Hesham El-Rewini \\ Department of \\ Computer Science and \\ Engineering \\ SMU, Dallas, TX, 75275- \\ 0122, USA. \\ 214-768-4309 \\ rewini@engr.smu.edu
}

\author{
Manal Houri \\ Department of \\ Computer Science and \\ Engineering \\ SMU, Dallas, TX, 75275- \\ 0122, USA. \\ 214-768-1673 \\ mhouri@engr.smu.edu
}

\begin{abstract}
In this paper, deployment of heterogeneous sensors in a field with preferential areas is studied. The problem is formulated using a mathematical program and solved optimally with an objective function that maximizes the coverage of the monitored field. The formulation considers several operation capabilities of the sensing devices including reliability, mobility, mobility cost, lifespan and power self-scheduling, as well as fields with preferential areas. For large-scale problems, a two-phase approach is proposed. A set of deployment patterns is first generated; and then assigned to the available devices considering their limited operational capabilities. Different sets of conducted experiments demonstrate the benefits of using heterogeneous sensors and fields with special monitoring requirements. In addition, the results show that the two-phase approach is capable of producing near-optimal coverage performance in a much shorter running time.
\end{abstract}

\section{Keywords}

Heterogeneity, sensor deployment, mobile sensors, mathematical programming, optimal solution.

\section{INTRODUCTION}

With the recent advances in sensing technology, smart sensors are introduced. These sensors have the capability to sense, compute, and be mobile. They can also form wireless sensor networks (WSN) that are usually used to monitor critical infrastructures such as transportation networks, border and building security, and air traffic control [29]. These infrastructures have distinct characteristics that may involve special monitoring. The components of the infrastructure itself may differ in their surveillance/security requirements. For instance, it is critical to monitor exits on the highways using reliable sensors during the rush hours while it is not as important in the early morning when there is barely any traffic.

Exploiting the heterogeneity of the sensing devices and considering the preferential areas in the monitored field may have a noticeable impact on the performance of the sensor networks. For example, mobile sensors could be used to cover the blind areas in the monitored fields. Sensors with state-switching capabilities could be turned off temporarily saving their energy to monitor important areas in the future. Using reliable sensors to cover the most critical areas will improve the security of the monitored field. Nonetheless, most of the sensor networks are used to monitor a field for a certain amount of time. Considering such feature will certainly affect sensors' organization and movement in the monitored field. However, the majority of the current research focuses on the deployment of homogenous sensors on a field with no preferential areas.

In this paper, we investigate the utilization of some of the sensing devices capabilities during the deployment process for coverage purpose. Coverage, in most of the literature, is defined as a measure of the quality of service of a sensors' sensing function. Our definition of coverage is slightly different. We define it as a measure of the security units that the sensing devices can collect in a certain time (horizon). We mainly focus on the mobility, movement cost, state-switching, sensors lifetime, and reliability of the sensing devices. Further, we study the effectiveness of using these sensors in a field with preferential areas which is closer to the reality of most of the sensor network applications. Our solution to the deployment problem includes two approaches. In the first approach, the optimal solution is developed by implementing a mathematical formulation to the problem. In the second, we apply a two-phase approach inspired from the vehicle routing problem solutions (VRP). The first approach guarantees the optimal solution to the deployment problem whereas the second is a greedy heuristic. An extensive set of experiments are conducted to study the performance of both approaches.

The rest of the paper is organized as follows. In the next section, related work is presented; in section 3, the problem is formally defined. Section 4 presents the optimal solution to the deployment problem; the two-phase approach is explained in section 5; the experimental results are depicted in section 6; finally the paper concludes in section 7 .

\section{RELATED WORK}

An early contribution to the problem of sensor deployment was by Chvatal in [10], who introduced the Art Gallery problem. In this problem, the goal was to determine the minimum number of observers required to secure an art gallery with a non-uniform geometry. Different versions of this problem have been studied to include mobile guards and guards with limited visibility (e.g. [30]). Nonetheless, research in the area of sensor deployment has rapidly advanced with the emergence of wireless sensor networks. Most of the work in this area concentrates on the study of the 
optimal formation of a wireless sensor network that can be used to collect data from a given field and to transmit it to one or more sink points. For example, Chakrabarty et al. [7] proposed a mathematical model for sensor and target location in distributed sensor networks. The formulation assumes homogenous sensing devices with perfect accuracy. Isler et al. [19] proposed concurrent and incremental deployment methods using the sampling theory in which new nodes are deployed based on samples taken from some other randomly deployed nodes.

Recently, the use of heterogeneous sensors attracted many researchers such as Mainwaring et al. [25] and Mhatre et al. [27] who provided real examples on heterogeneous sensors' utilization. In addition, Gupta et al. [17] demonstrated that homogenous networks suffer from poor scalability. These examples show the importance of heterogeneity in terms of data forwarding, processing, and sensor network lifetime. Other researchers focused on other perspectives of sensors heterogeneity. For example, in [28], the authors studied the optimal deployment of nodes with different initial energy levels that minimizes the sensor network energy cost using different communication modes, namely single-hop and multi-hop. Kumar et al. [21] explored the advantage of using heterogeneous sensors in a hierarchical network. The network is structured based on the sensors computational capabilities. The results show that using structured sensors with different computational capabilities outperforms homogenous networks with approximately the same energy budget.

The effect of heterogeneity on the sensor network lifetime has been studied in [18], [23], and [24]. [23] mathematically analyzed the effect of deploying sensors with different energy capabilities on the overall sensor network lifetime. Highly capable sensors are used as cluster heads that collect and process the data received from less capable sensors. This combination of sensors enhances the duration of the network sensing operation. [24] proposed an integer linear program to maximize the overall lifetime of wireless sensor networks. Nodes are forced to send collected data as far as they could and bypass some intermediate nodes to save their energy. [18] proposed the deployment of a superior set of sensors, called microservers in a hybrid deployment framework. These microservers are used to filter and route the data in order to reduce the load on other devices.

Sensors state-switching capability is mainly utilized by power saving techniques and routing protocols as in, [32], [33], [38], [39], and [40]. [40] introduced S-MAC which is a protocol developed to address energy issues in sensor networks. It is based on a simple scheduling scheme that allows neighbors to sleep for long periods and synchronize wakeups. [32] proposed a two-radio architecture, STEM, that saves energy by letting the data radio sleep until communication is required. In the mean time, the other radio (wakeup) periodically listens using low duty cycles. In GAF [38], the network is divided into fixed grids using location information where nodes in each grid alternate between sleeping and listening. However, these wakeup schemes may affect the communication delay of sensor network (for example see [39]). Subramanian in [33] analytically proved that sleep scheduling maximizes the lifetime of sensor networks by $O\left(\sqrt{\frac{n}{\log n}}\right.$, where $n$ is the number of sensors in the network.
Deployment of mobile sensors has been described in several contexts. One common approach assumes availability of a superior leader that guides several mobile sensor nodes to their deployment positions. [36] proposed an algorithm that moves mobile nodes to cover the blind spots in the monitored area based on static nodes bidding data. A similar algorithm that exploits the mobile sensors in covering the blind areas in the monitored field is introduced by Wang et al in [35]. The algorithm uses Voronoi diagram to discover the blind spots and move the nearest mobile sensor to cover them. In addition to mobility, issues related to sensors' reliability are presented in [2] and [14]. They consider the deployment of stationary sensing devices with imprecise detection capabilities. The objective is to maximize field coverage for target detection using a set of devices with probabilistic precessions.

One common drawback of the previous deployment methodologies is that they ignore the collective representation of the advanced capabilities (e.g., power state-switching and mobility) and operational limitations of the sensors (e.g., lifespan limitation, mobility cost, and reliability degradation). Furthermore, most of these approaches assume static network conditions over the monitoring horizon. In other words, security requirements of the monitored fields are assumed to be constant, which contradicts the realistic representation of the monitored fields. In this paper, our work considers the majority of these requirements during the deployment process.

\section{PROPLEM STATEMENT}

A deployment field $F(A)$ with differential security requirements is to be monitored for a time horizon of length $T$. In addition, a set of heterogeneous sensors $|S|$ is given. These sensors differ in their capabilities such as lifespan/lifetime, allowed number of stateswitching, allowed number of moves, movement cost, and reliability. The objectives are to achieve the maximum coverage of the monitored field, exploit sensors' capabilities, prolong sensor network lifetime, and increase the security of the monitored field. The latter is enhanced by using the highest reliable sensors on the most important areas in the field. The deployment problem is modeled as follows:

The monitored field is divided into a number of square-shaped zones $|A|$. Each zone $i \in A$ is associated with a time-varying weight function $w_{i}^{t}$, where $t \in T$. This weight function defines the importance of the observations (surveillance requirement) in this zone over the horizon $T$. A sensor lifespan $L_{s}$ is the number of time units that the sensor was used to monitor one or more zones of the monitored field. Each sensor lifespan is associated with a cost $e_{s}$. In addition to lifespan, sensors are assumed to have an allowed number of state-switching $P_{s}$ in which a sensor $s \in S$ can change its state from "on" to "off" or vice versa based on the field requirements. For instance, a sensor $s \in S$ could be switched to "off" at time $t \in T$ to save its lifetime for a different zone with a higher security requirement $w_{i}^{t}$ at different times.

Moreover, a sensing device could be stationary or mobile. If a stationary device is deployed on a zone $i \in A$, this device is assumed to remain in the center of this zone for its entire lifespan. On the contrary, a mobile sensor can cover multiple zones over a 
time period $T$. All mobile devices are assumed to have no restrictions on the start or the end locations of their deployment, but they have restrictions, $M_{\mathrm{s}}$, per sensor on the number of moves from zone to another. A sensor transfer between two zones is assumed to be associated with a cost. This cost is expressed in terms of the loss in the device lifespan $E_{s i j}^{t}$. Nevertheless, each sensor $s \in S$ is characterized by a predefined reliability $R_{s}^{t}$ that typically changes over time.

Considering the heterogeneity of sensing devices and the differentiated security requirements, an optimal deployment scheme is required. The scheme should exploit the sensors'capabilities as well as cover the most important areas of the monitored field.

\section{OPTIMAL SOLUTION}

The deployment problem is formulated in the form of an integer mathematical program. A detailed version of the program can be found in our previous work [31].

\section{Program A:}

Maximize: $\sum_{t} \sum_{i} \sum_{s} w_{i}^{t} \cdot x_{s i}^{t} \cdot R_{s}^{t}$

Subject to:

Deployment Constraints

$$
\begin{aligned}
& x_{s i}^{t}+y_{s i}^{t} \leq 1 \quad \forall t, i, s \\
& y_{s i}^{t+1} \geq x_{s i}^{t}-\sum_{j} x_{s j}^{t+1} \quad \forall t, i, s \\
& y_{s i}^{t-1} \geq x_{s i}^{t}-\sum_{j} x_{s j}^{t-1} \quad \forall t, i, s \\
& y_{s i}^{t+1} \geq y_{s i}^{t}-\sum_{j} x_{s j}^{t+1} \quad \forall t, i, s \\
& y_{s i}^{t-1} \geq y_{s i}^{t}-\sum_{j} x_{s j}^{t-1} \quad \forall t, i, s \\
& \text { Assignment Constraints } \\
& \begin{array}{ll}
\sum_{i}\left(x_{s i}^{t}+y_{s i}^{t}\right) \leq 1 & \forall t, s \\
\sum_{s} x_{s i}^{t} \leq 1 & \forall t, i
\end{array} \\
& \text { Mobility Constraints } \\
& m_{s i j}^{t} \geq\left(\left(x_{s j}^{t+1}+y_{s j}^{t+1}\right)+\left(x_{s i}^{t}+y_{s i}^{t}\right)\right)-1 \quad \forall t, i, j, i \neq j, s \\
& m_{s i j}^{t} \leq x_{s j}^{t+1}+y_{s j}^{t+1} \quad \forall t, i, j, s \\
& m_{s i j}^{t} \leq x_{s i}^{t}+y_{s i}^{t} \quad \forall t, i, j, s \\
& \sum_{i} \sum_{j} \sum_{t} m_{s i j}^{t} \leq M_{s} \quad \forall s \\
& \text { State Switching Constraints } \\
& o n_{s i}^{t} \geq\left(x_{s i}^{t+1}+y_{s i}^{t}\right)-1 \quad \forall t, i, s \\
& o n_{s i}^{t} \leq x_{s i}^{t+1} \quad \forall t, i, s \\
& o n_{s i}^{t} \leq y_{s i}^{t} \quad \forall t, i, s
\end{aligned}
$$

$$
\begin{array}{ll}
o f f_{s i}^{t} \geq\left(y_{s i}^{t+1}+x_{s i}^{t}\right)-1 & \forall t, i, s \\
o f f_{s i}^{t} \leq y_{s i}^{t+1} & \forall t, i, s \\
o f f_{s i}^{t} \leq x_{s i}^{t} & \forall t, i, s \\
\sum_{t} \sum_{i}\left(o n_{s i}^{t}+o f f_{s i}^{t}\right) \leq P_{s} & \forall s
\end{array}
$$

Lifespan Constraints

$\sum_{t} \sum_{i} e_{s} x_{s i}^{t}+\sum_{i} \sum_{j} \sum_{t} E_{s i j}^{t} m_{s i j}^{t} \leq L_{s} \quad \forall s$

Binary Constraints

$$
\left\{x_{s i}^{t}, y_{s i}^{t}, m_{s i j}^{t}, o n_{s i}^{t}, o f f_{s i}^{t}\right\}=1 \text { or } 0 \quad \forall t, i, s
$$

As shown in (1), the objective function maximizes the field coverage which is described as the sum over all time intervals, zones and sensing devices of the products of the observation weight $w_{i}^{t}$, the decision variable $x_{s i}^{t} \quad\left(x_{s i}^{t}=1\right.$, if device $s$ exists in an active state on zone $i$ in time interval $t$ ) and the reliability of the used device $R_{s}^{t}$. Constraint (2) ensures that a sensing device is either active or inactive during any time interval. Constraints (3) to (6) determine the value of the binary variable $y_{s i}^{t}$ based on the value of $x_{s i}^{t}$. Constraint (7) ensures that each zone is covered by at most one device in any time interval. Also, at each time interval, a sensing device is covering at most one zone, which is guaranteed in constraint (8). Constraints (9) to (11) determine if a sensing device $s \in S$ is moved from zone $i$ to zone $j$ at the end of interval $t$. Constraint (12) ensures that the number of moves made by a device $s \in S$ is less than or equal to the maximum allowed number of moves for this device.

The state-switching of a sensing device $s \in S$ from active to inactive and vice versa is determined in constraints (13) to (18). The total number of state-switching for each sensing device is computed and compared to the allowed maximum number of switching for each device as given in constraint (19). Constraint (20) ensures that each sensing device is not utilized beyond its lifespan. The consumption of a device's lifespan is computed as the sum over all intervals in which the device is active plus the loss in the device lifespan associated with its moves on the different zones. Finally, the integrality of all binary variables is preserved in constraint (21).

The average results over many experiments using this mathematical formulation showed that the problem is intractable and the optimal solution can be used only in small-scale problems. However, this solution helped in understanding the characteristics of the deployment problem and it is also used as a baseline to the two-phase approach in small-scale problems.

\section{TWO-PHASE APPROACH}

A simplified version of the deployment problem could be viewed as one version of the Vehicle Routing Problem (VRP). According to the definition of VRP, vehicles are routed to serve a list of customers while optimizing one or more predefined objectives (e.g., routing cost, number of covered customers, generated revenue, etc). This problem could involve constraints on different 
capacity vehicles, traveling distance, driving and unloading times, and customer delivery time windows, etc. [22]. We are interested in the deterministic version of the VRP where customers' demand and travel cost are known a priori. Several formulations and solution algorithms are given to this problem [34]. Nevertheless, an optimal solution using these algorithms could be obtained only for small-scale problems [15]. This initiates devising fast heuristics that achieve acceptable near optimal solutions. One of the best-known heuristics for the VRP is the two-phase approach [11]. As its name implies, it consists of two phases. The first phase includes clustering customers by assigning them to vehicles. The second phase generates an efficient route for each vehicle.

Our solution to the deployment problem is inspired by the VRP two-phase approach. In the first phase, a deployment pattern for each sensor is generated assuming that sensors don't have any limitations in terms of lifetime, state-switching, or mobility. Then, in the second phase, the generated patterns are matched with the sensors capabilities. In addition, an improvement algorithm is developed to enhance the final deployment scheme.

The idea of the two-phase approach is to decompose the problem in terms of the available set of sensing devices. The number of the sensing devices is assumed to be less than or equal to the number of zones. Observations are clustered in deployment patterns. Each pattern is described in terms of its zones and time intervals during which these zones are visited. These patterns are not overlapping in the sense that two patterns cannot include the same observation. These patterns are also generated for hypothetical sensing devices with unlimited lifespan, unconstrained mobility, and unrestricted state-switching capabilities. Available sensing devices are then assigned to these patterns. In this step, an optimal matching problem is solved to ensure that the available devices are optimally utilized. Using the two-phase approach, a solution algorithm is developed. Using the two-phase approach, a solution algorithm is developed. The algorithm consists of two main phases as follows.

Phase 1: Generation of development patterns

Phase 2: assigning sensors to the generated patterns

Step 1: Determining device-pattern performances

Step 2: Device-pattern matching

Step 3: Solution improvement strategy

\section{Phase 1: Generation of deployment patterns}

In the first phase, we generate the deployment patterns for a set of hypothetical devices $S^{\prime}$ with unlimited capabilities. The size of this set $|k|$ is equal to the number of available devices for deployment. However, as illustrated in Figure 1, one can also try to generate more deployment patterns than the number of available devices, but the experiments in that case show that the coverage performance decreases.

Three types of patterns are generated, 1) Highest observation Patterns (HP), 2) Reliable Patterns (RP), and 3) low Movement cost Patterns (MP). These patterns are generated to exploit different parameters of the sensing devices as well as the heterogeneity of the deployment field.

In HP, patterns are generated through solving the mathematical program given in $(B)$. The formulation in $(B)$ solves a generalized assignment problem with the objective being to generate $|k|$ deployment patterns with maximum observation weights. Two constraints are considered in the program; constraint (a2) ensures that at any time interval each sensing device is deployed only on one zone. In addition, each zone is covered by only one device at a time, which is guaranteed in constraint (a3).

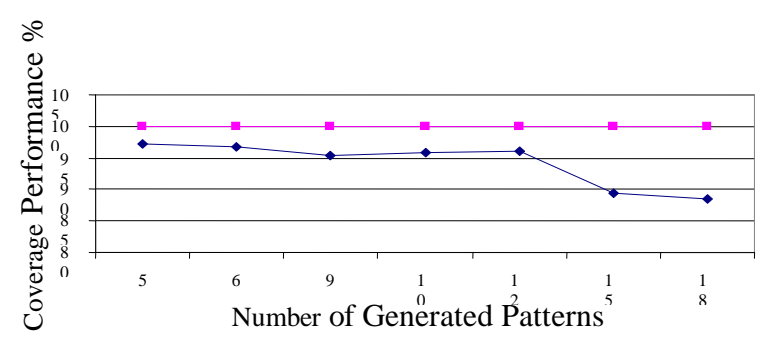

Figure 1: Effect of the number of generated patterns on coverage performance.

\section{Program B:}

$\begin{array}{ll}\text { Maximize: } \sum_{t} \sum_{i} \sum_{s^{\prime}} w_{i}^{t} \cdot x_{s i}^{t} & \\ \sum_{i} x_{s^{\prime} i}^{t} \leq 1 & \forall t, s^{\prime} \\ \sum_{s^{\prime}} x_{s^{\prime} i}^{t} \leq 1 & \forall t, i \\ x_{s^{\prime} i}^{t}=1 \text { or } 0 & \forall t, i, s^{\prime}\end{array}$

In RP, at each time interval, the highest observations are assigned to sensors with the highest reliability. As given in program (C), the objective function is modified to include sensors' reliability.

Program C:

$\begin{array}{cc}\text { Maximize: } \sum_{t} \sum_{i} \sum_{s} w_{i}^{t} \cdot x_{s i}^{t} \cdot R_{s}^{t} \\ \sum_{s} x_{s i}^{t} \leq 1 \\ \sum_{i} x_{s i}^{t} \leq 1 & \forall t, s \\ \forall t, i\end{array}$

Binary Constraints $x_{s i}^{t}=1$ or $0 \forall t, i, s$

Zones in MP are clustered in the different patterns such that the sensors' total transfer cost is minimized. Program (D) is used to generate these patterns in which the movement cost is considered as part of the objective function as given in constraint (a9). Some of the optimal program constraints given in program $(A)$ are used in this program such as deployment, assignment, and mobility without limitations on the number of moves.

\section{Program D:}

Maximize: $\sum_{t} \sum_{i} \sum_{s} w_{i}^{t} \cdot x_{s i}^{t}-m_{s i j}^{t} * \operatorname{Cos} t_{s i j}^{t}$ (a9)

Deployment Constraints

$x_{s i}^{t}+y_{s i}^{t} \leq 1 \quad \forall t, i, s$

(a10) 


$$
\begin{aligned}
& y_{s i}^{t+1} \geq x_{s i}^{t}-\sum_{j} x_{s j}^{t+1} \quad \forall t, i, s \quad \text { (all) } \\
& y_{s i}^{t-1} \geq x_{s i}^{t}-\sum x_{j s}^{t-1} \quad \forall t, i, s \quad \text { (al2) } \\
& y_{s i}^{t+1} \geq y_{s i}^{t}-\sum_{j} x_{j s}^{t+1} \quad \forall t, i, s \quad \text { (al3) } \\
& y_{s i}^{t-1} \geq y_{s i}^{t}-\sum_{j} x_{j s}^{t-1} \quad \forall t, i, s \quad \text { (al4) } \\
& \begin{array}{l}
\text { Assignment Constraints } \\
\sum\left(x_{s i}^{t}+y_{s i}^{t}\right) \leq 1 \quad \forall t, s \quad \text { (a15) }
\end{array} \\
& \sum_{s} x_{s i}^{t} \leq 1 \quad \forall t, i \\
& m_{s i j}^{t} \geq\left(\left(x_{s j}^{t+1}+y_{s j}^{t+1}\right)+\left(x_{s i}^{t}+y_{s i}^{t}\right)\right)-1 \quad \forall t, i, j, i \neq j, s \quad(a 17) \\
& m_{s i j}^{t} \leq x_{s j}^{t+1}+y_{s j}^{t+1} \quad \forall t, i, j, s \\
& m_{s i j}^{t} \leq x_{s i}^{t}+y_{s i}^{t} \quad \forall t, i, j, s \\
& \left\{x_{s i}^{t}, y_{s i}^{t}, m_{s i j}^{t}\right\}=1 \text { or } 0 \quad \forall t, i, s
\end{aligned}
$$

Programs B, C, and D could also be modified to generate patterns with a limited number of moves. As given in (a22), the total number of moves is added to the objective function with negative signs and user-specific weight parameter $\mu$. Increasing the value of $\mu$ results in more restrictive patterns in terms of the number of moves.

$$
\text { Maximize: } \sum_{t} \sum_{i} \sum_{s^{\prime}} w_{i}^{t} \cdot x_{s^{\prime} i}^{t}-\mu . \sum_{t} \sum_{i} \sum_{j} \sum_{s^{\prime}} m_{s^{\prime} i j}^{t}
$$

\section{Phase 2: Assigning sensors to the generated patterns}

\section{Step 1: Determining device-pattern performances}

For the general problem, deployment patterns generated in phase 1 could be different in terms of the sum of the weights of their observations. In addition, they might require a different number of moves by the sensing device. Also, there is no control on how observations are distributed among these patterns. As such, sensing devices with different operational characteristics $\left(R_{s}^{t}, L_{s}, M_{s}, P_{s}\right.$ and $\left.E_{s i j}^{t}\right)$ are expected to result in different performances when assigned to each of these deployment patterns. Step 1 determines the performance of each sensing device when assigned to each of the generated patterns. This is done through determining the objective function of the mathematical program given in $(E)$. Program $(E)$ determines the maximum achievable coverage performance when one sensing device with certain operational capabilities is assigned to one deployment pattern.

Assigning a sensing device with different parameters to one of the generated deployment patterns may produce a different deployment scheme. Sensors are defined using their reliability level $R^{t}$, lifespan $L$, maximum allowed moves $M$, lifespan loss of $E_{i j}^{t}$ associated with each move, and maximum allowed stateswitching $P$.

On the other hand, the deployment pattern is defined using the weight parameter $\bar{w}_{i}^{t}$, which is equal to $w_{i}^{t}$ if the observation on zone $i$ in time interval $t$ is included in this pattern, and zero otherwise. A pattern is also described by the subset of zones $A^{\prime}$ included in this pattern. The decision variable $x_{i}^{t}$ is set to 1 if observation $\bar{w}_{i}^{t}$ remains in the pattern and zero otherwise. We also use the descriptive variables $y_{i}^{t}, m_{i j}^{t}, O n_{i}^{t}$ and $O f f_{i}^{t}$ which are the same that were used in the formulation of the main problem (program $A$ ). Program $E$ is very similar to the formulation of program $A$ with the exception that the problem is solved for only one device. Also, this device is allowed to move on the subset $A^{\prime}$ of the field zones. The objective function and the list of constraints for program $(E)$ are given below.

\section{Program E:}

Maximize $\sum_{t} \sum_{i \in A^{\prime}} \bar{w}_{i}^{t} \cdot x_{i}^{t} \cdot R^{t}$

Subject to:

Deployment Constraints

$\begin{array}{ll}x_{i}^{t}+y_{i}^{t} \leq 1 & \forall t, i \in A^{\prime} \\ y_{i}^{t+1} \geq x_{i}^{t}-\sum_{j \in A^{\prime}} x_{j}^{t+1} & \forall t, i \in A^{\prime} \\ y_{i}^{t-1} \geq x_{i}^{t}-\sum_{j \in A^{\prime}} x_{j}^{t-1} & \forall t, i \in A^{\prime} \\ y_{i}^{t+1} \geq y_{i}^{t}-\sum_{j \in A^{\prime}} x_{j}^{t+1} & \forall t, i \in A^{\prime} \\ y_{i}^{t-1} \geq y_{i}^{t}-\sum_{j \in A^{\prime}} x_{j}^{t-1} & \forall t, i \\ \text { Assignment Constraints } & \\ \sum_{j \in A^{\prime}}\left(x_{i}^{t}+y_{i}^{t}\right) \leq 1 & \forall t\end{array}$

Mobility Constraints

$m_{i j}^{t} \geq\left(\left(x_{j}^{t+1}+y_{j}^{t+1}\right)+\left(x_{i}^{t}+y_{i}^{t}\right)\right)-1$ $\forall t, i \in A^{\prime}, j \in A^{\prime}, i \neq j$

$m_{i j}^{t} \leq x_{j}^{t+1}+y_{j}^{t+1}$

$\forall t, i \in A^{\prime}, j \in A^{\prime}$

$m_{i j}^{t} \leq x_{i}^{t}+y_{i}^{t}$

$\forall t, i \in A^{\prime}, j \in A^{\prime}$

$\sum_{i \in A^{\prime}} \sum_{j \in A^{\prime}} \sum_{t} m_{i j}^{t} \leq M$

State Switching Constraints

$O n_{i}^{t} \geq\left(x_{i}^{t+1}+y_{i}^{t}\right)-1 \quad \forall t, i \in A^{\prime}$

$O n_{i}^{t} \leq x_{i}^{t+1}$

$\forall t, i \in A^{\prime}$

$O n_{i}^{t} \leq y_{i}^{t}$

$\forall t, i \in A^{\prime}$

$O f f_{i}^{t} \geq\left(y_{i}^{t+1}+x_{i}^{t}\right)-1$

$\forall t, i \in A^{\prime}$

$O f f_{i}^{t} \leq y_{i}^{t+1}$

$\forall t, i \in A^{\prime}$

(b16) 


$$
\begin{aligned}
& O f f_{s i}^{t} \leq x_{s i}^{t} \quad \forall t, i \in A^{\prime} \\
& \sum_{t} \sum_{i \in A^{\prime}}\left(O n_{i}^{t}+O f f_{i}^{t}\right) \leq P \quad \forall t, i \in A^{\prime} \\
& \text { Lifespan Constraints } \\
& \sum_{t} \sum_{i \in A^{\prime}} x_{i}^{t}+\sum_{i \in A^{\prime}} \sum_{j \in A^{\prime}} \sum_{t} E_{i j}^{t} m_{i j}^{t} \leq L \quad \forall t, i \in A^{\prime} \\
& \text { Binary Constraints } \\
& \left\{x_{i}^{t}, y_{i}^{t}, m_{i j}^{t}, O n_{i}^{t}, O f f_{i}^{t}\right\}=1 \text { or } 0 \quad \forall t, i \in A^{\prime}
\end{aligned}
$$

\section{Step 2: Device-pattern matching}

The objective function of program $(E)$ gives the total observation weights $C_{s p}$ that could be collected by assigning a sensing device $s \in S$ to a deployment pattern $p \in P$ considering the limited capability of this device. Now, assume that this value is determined for all device-pattern combinations. The problem is to find the optimal match between available devices and the deployment patterns such that available devices are optimally utilized. The optimal device-pattern matching scheme is determined by solving an assignment problem using the mathematical program given in $(F) . V_{s p}$ is equal to 1 if device $s \in S$ is assigned to pattern $p$, and zero otherwise. The objective function of this program, given in $(c 1)$, maximizes the weights of collected observations. Each deployment pattern is covered by only one device and each device is used only once as given by constraint (c2) and constraint (c3), respectively.

\section{Program F:}

Maximize: $\sum_{s} \sum_{p} C_{s p} . V_{s p}$

Subject to:

$$
\begin{array}{ll}
\sum_{s} V_{s p} \leq 1 & \forall s, p \\
\sum_{p} V_{s p} \leq 1 & \forall s, p
\end{array}
$$

\section{Step 3: Solution improvement strategy}

The last step of the solution algorithm implements a fast improvement strategy to the solution obtained so far. The idea behind this strategy is to swap low weight observations in the deployment pattern of high reliability sensors with high weight observations of other low reliability sensors, if any. The two swapped observations must coincide in the same time interval. Thus, as shown in Figure 2, if two observations with weight difference $\Delta w$, the resulting improvement in the overall coverage performance is $\Delta w . \Delta R$, where $\Delta R$ is the reliability difference between the two sensors. However, swapping two observations could result in a different number of moves of the sensors. It could also result in a different lifespan loss. Therefore, all new deployment patterns have to be checked for feasibility in terms of mobility and lifespan. The steps used to implement this improvement strategy are as follows:

Step 3a: Find the unmarked observations with the highest $\Delta w . \Delta R$. Step $3 b$ : Check the feasibility of these swapping observations.
Step 3c: If feasible, update the deployment patterns for the two sensors. Otherwise, mark this swapping step as infeasible and go to Step 3a.

Step 3d: If no more observations with positive swap exist, stop and output the new deployment patterns; otherwise, go to step 3a.

Although this algorithm is very simple, the experiments show that the average improvement in the coverage performance is up to $4 \%$ of the overall coverage.

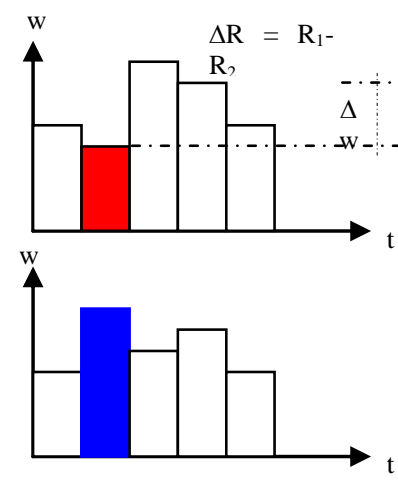

Before Observation Swapping

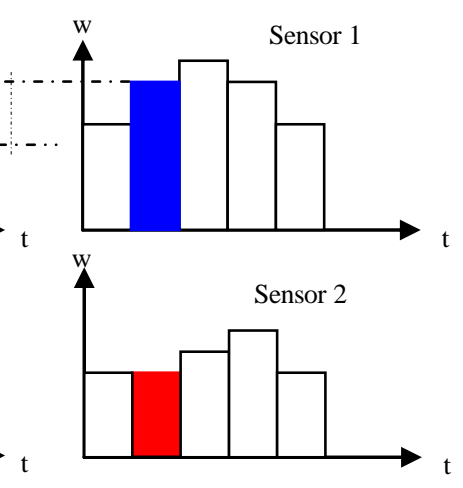

After Observation Swapping
Figure 2: Example of observation swapping

\section{EXPERIMENTAL RESULTS}

In the following subsections, the performance of the optimal solution and two-phase approach is reported. Then, the twophase approach is compared to the optimal solution results (Program $A$ ) for small-scale problems. Other several experiments are also conducted for large-scale problems.

\subsection{Optimal Solution Limitations}

In this section, we report our experience dealing with the optimal solution of the deployment problem. We implemented our formulation using a commercial version of CPLEX 8.0 with AMPL interface. The used optimization package (CPLEX 8.0) runs on a $2.4 \mathrm{GHz}$ machine with $2 \mathrm{~GB}$ memory. This optimal solution is also used to benchmark the performance of the twophase algorithm in the next sections.

Three different sets of experiments are conducted. These experiments study the effect of increasing the number of zones, number of sensors, and time horizon on the running time required to generate the optimal solution, respectively. As shown in Table 1 , the experiments are classified in three categories. In the first category (experiments 1-4), we study the effect of increasing the number of sensors from 10 to 35 on the solution performance, while the number of sensors and the horizon are set to 5 and 12 respectively. In the second category, the number of sensors changes from 3 to 15 , while the number of zones and the horizon are set to 20 and 5, respectively. In the final category, the horizon is increased from 2 to 25 units of time. In this category, the number of zones and sensors are set to 20 and 5, respectively.

In all the experiments, the time-varying observations of different zones were generated randomly following a uniform distribution $U(0,200)$. In addition, a heterogeneous set of sensors is assumed.

The sensors' lifespan $L_{s}$ is generated randomly as a function of the length of the monitoring horizon, while $M_{S}$ and $P_{S}$ are 
generated randomly based on $L_{s}$. For example, if the monitoring horizon is $T$ intervals, the sensor lifespan is generated randomly using the uniform distribution $U(1, T)$ and both $M_{s}$ and $P_{s}$ use a uniform distribution function $U\left(1, L_{s}\right)$. In addition, sensors reliability $R_{s}^{t}$ is generated randomly using a uniform random generator $R(0,1)$, where 0 and 1 represent $0 \%$ and $100 \%$ reliability, respectively. Furthermore, the lifespan cost $e_{s}$ is set to unity throughout these experiments.

Table 1 reports the average results of the optimal solution over multiple runs. As shown, the running time required to generate the optimal solution increases exponentially with the increase of the problem size. For instance, a running time of 1960 seconds is recorded for a problem of 10 zones, 5 sensors and a horizon of 12 intervals. This running time jumps to 39670 seconds when the number of zones is increased to 25. Problem settings with dimensions beyond the ones presented in the table could not be generated using the machine mentioned above.

Table 1: Global optimal solution for different problem settings

\begin{tabular}{|c|c|c|c|c|c|}
\hline $\begin{array}{c}\text { Exp } \\
\text { No. }\end{array}$ & $\begin{array}{c}\text { No. of } \\
\text { Zones }\end{array}$ & $\begin{array}{c}\text { No. of } \\
\text { sensors }\end{array}$ & Horizon & Objective & $\begin{array}{c}\text { Running } \\
\text { Time (s) }\end{array}$ \\
\hline 1 & 10 & 5 & 12 & 940 & 1960 \\
2 & 20 & 5 & 12 & 990 & 32030.8 \\
3 & 25 & 5 & 12 & 961 & 39670 \\
4 & 35 & 5 & 12 & NA & NA \\
\hline 5 & 20 & 3 & 12 & 480 & 2400 \\
6 & 20 & 5 & 12 & 990 & 32030.8 \\
7 & 20 & 10 & 12 & 2000 & 50056 \\
8 & 20 & 15 & 12 & NA & NA \\
\hline 9 & 20 & 5 & 3 & 670 & 300.8 \\
10 & 20 & 5 & 6 & 860 & 2300 \\
11 & 20 & 5 & 12 & 990 & 32030.8 \\
12 & 20 & 5 & 24 & NA & NA \\
\hline
\end{tabular}

\subsection{Two-Phase Solution Performance}

In this section, the implementation (using the commercial version of ILOG CPLEX) of the two-phase approach is evaluated. Table 2 shows the objective performance and running time as a percentage from the global optimal solution results. The results show that as the number of zones increases, HP, RP, and MP patterns performances are almost in a fixed range from the global optimal performance. Despite that, HP patterns have the best objective performance among other patterns; their performance is almost $93 \%$ of the global optimal. When the number of sensors changes, all the patterns seem to perform equally. The average performance gained in this case is $95 \%$. As the horizon time increases, HP has an objective performance equal to $97 \%$ from the global optimal performance. From the running time perspective, the two-phase approach is taking in average $55 \%$ of the total time when small networks are considered. However, MP takes more time than other patterns. In conclusion, Table 2 shows that HP has the best average performance with different problem settings.

\subsection{A Comparison between the Two-Phase Approach Patterns}

In previous sections, many experiments with different problem settings were conducted to show the objective performance and running time for small-scale problems. In this section, a large set of experiments is conducted to study the performance of the generated patterns using the two-phase approach compared to each other. In these experiments, a range of 20 to 100 zones are monitored by 50 to 800 heterogeneous sensors for 12 units of time. Sensors lifetime, state-switching, and mobility are randomly generated. In addition, sensors reliability is generated randomly within the range of [0\%-100\%]. Moreover, zones weights are uniformly generated within a range of [0-200] security units.

Figure 3 shows the average objective performance of the twophase approach. The results, in general, show no major difference among the patterns objective performances. However, the running time of the MP, as shown in Figure 4, is much larger than the running time of the HP and RP. Another observation is that RP on average has the least running time of all patterns, while their objective performance is almost equal to the HP. The results mainly suggest using RP patterns where the values of the sensors parameters are uniformly distributed.

\section{CONCLUSION}

In this paper, we showed the effect of the sensors heterogeneity as well as the monitored field differential security requirements on the overall coverage. The problem is stated as a deployment of a heterogeneous set of sensing devices to maximize the coverage of a field with differential security service requirements. An integer mathematical program, with the objective of maximizing the overall network coverage, was presented. The formulation considered several operation characteristics for the sensing devices including reliability, mobility, mobility cost, lifespan, and power self-scheduling. Using the two-phase approach, a list of deployment patterns is first generated. These patterns are then assigned to the available devices. The approach also applies a fast heuristic strategy to improve the solution quality obtained so far. A set of experiments was conducted to test the solutions' performance for different problem settings. The two-phase approach on average reduces the running time of the optimal solution by $50 \%$ while losing $8 \%$ of the coverage performance which is a reasonable price compared to the objective gain.

Several extensions are considered for the research work presented in this paper. For example, given the uncertainty associated with security requirement in most sensing operations, the stochastic version of this problem could be considered. Another extension is to consider problems in which a portion of the sensing device is restricted only to conduct sensing tasks with specific properties. In addition, the main objective of the problem presented in this paper is to maximize overall network coverage. Another important objective in sensing operations is to be able to track the location of one or more moving objects. Developing a deployment scheme to achieve this objective could be another direction. Finally, providing the capability to incorporate real-time device deployment decisions is another important extension to this work. 
Table 2: Two-phase solution performance percentage

\begin{tabular}{|c|c|c|c|c|c|c|c|c|c|}
\hline $\begin{array}{l}\text { Exp } \\
\text { No. }\end{array}$ & $\begin{array}{c}\text { No. of } \\
\text { zones }\end{array}$ & $\begin{array}{c}\text { No. of } \\
\text { sensors }\end{array}$ & Horizon & \multicolumn{2}{|c|}{ HP } & \multicolumn{2}{|c|}{$\mathrm{RP}$} & \multicolumn{2}{|c|}{ MP } \\
\hline & & & & $\begin{array}{c}\text { Objective } \\
(\%)\end{array}$ & $\begin{array}{l}\text { Running } \\
\text { Time (\%) }\end{array}$ & $\begin{array}{c}\text { Objective } \\
(\%)\end{array}$ & $\begin{array}{c}\text { Running } \\
\text { Time (\%) }\end{array}$ & $\begin{array}{c}\text { Objective } \\
(\%)\end{array}$ & $\begin{array}{l}\text { Running } \\
\text { Time (\%) }\end{array}$ \\
\hline 1 & 10 & 5 & 12 & 94 & 45 & 87 & 44 & 91 & 56 \\
\hline 2 & 20 & 5 & 12 & 94 & 46 & 87 & 43 & 91 & 60 \\
\hline 3 & 25 & 5 & 12 & 94 & 48 & 89 & 49 & 80 & 62 \\
\hline 4 & 20 & 3 & 12 & 98 & 40 & 97 & 40 & 96 & 59 \\
\hline 5 & 20 & 5 & 12 & 94 & 46 & 87 & 43 & 91 & 60 \\
\hline 6 & 20 & 10 & 12 & 87 & 48 & 87 & 45 & 90 & 63 \\
\hline 7 & 20 & 5 & 3 & 99 & 40 & 81 & 41 & 96 & 50 \\
\hline 8 & 20 & 5 & 6 & 98 & 43 & 87 & 42 & 96 & 57 \\
\hline 9 & 20 & 5 & 12 & 94 & 46 & 87 & 43 & 91 & 60 \\
\hline
\end{tabular}

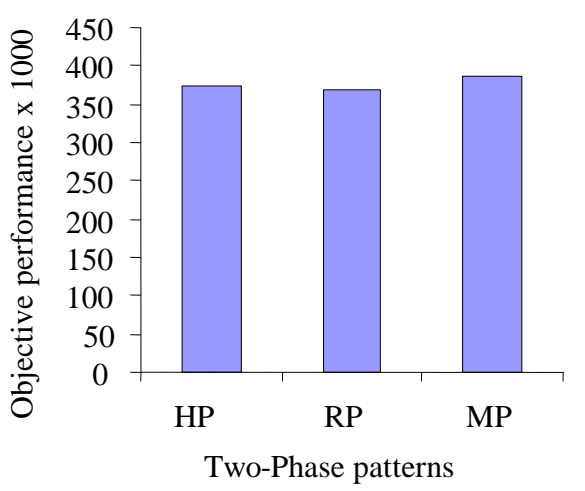

Figure 3: Objective performance for the proposed heuristics

\section{REFERENCES}

[1]. Agarwal, Y., Mathur, K., and Salkin, H. Set partitioning approach to vehicle routing. Networks, 7 (1989), 731-749.

[2]. Araque, J.R., Kudva, G., Morin, T., and Pekny, J. A branch-and-cut algorithm for vehicle routing problems. Annals of Operations Research, 50 (1994), 37-59.

[3]. Augerat, P., Belenguer, J., Benavent, E., Corber'an, A., Naddef, D., and Rinaldi, G. Computational results with a branch and cut code for the capacitated vehicle routing problem. Research Report 949-M, Université Joseph Fourier, Grenoble, France, 1995.

[4]. Balinski, M. and Quandt, R. On an integer program for a delivery problem. Operations Research, 12 (1964), 300304.

[5]. Blasum, U. and Hochstättler, W. Application of the branch and cut method to the vehicle routing problem. Technical Report zpr 2000-386, Zentrum für Angewandte Informatik, Koln, 2000.

[6]. Cardei M., and $\mathrm{Wu}, \mathrm{J}$. Coverage in wireless sensor networks. Handbook of Sensor Networks. Ilyas, M. and Mahgoub, I. (eds.). CRC Press, 2004.

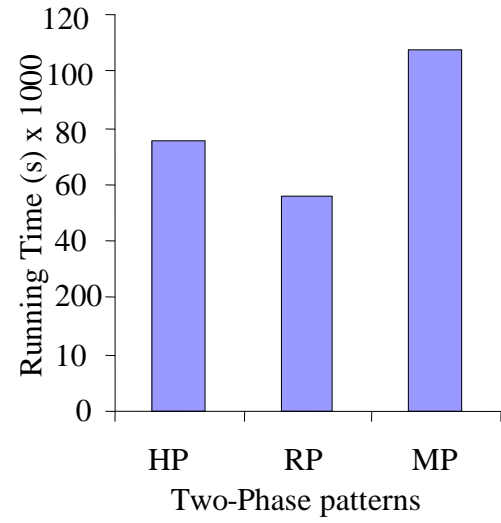

Figure 4: Running time for the proposed heuristics

[7]. Chakrabarty, K., Iyengar, S., Qi, H., and Cho, E. Grid coverage for surveillance and target location in distributed sensor networks. IEEE Transactions on Computers, 51 (2002), 1448-1453.

[8]. Christofides, N., and Eilon, S. An algorithm for the vehicle dispatching problem. Operational Research Quarterly, 20 (1969), 309-318.

[9]. Christofides, N., Mingozzi, A., and Toth, P. Exact algorithms for solving the vehicle routing problem based on spanning trees and shortest path relaxations. Mathematical Programming, 20 (1981), 255-282.

[10]. Chvatal, V. A combinatorial theorem in plane geometry. Journal of Computorial Theory (B), 18 (1975), 39-41.

[11]. Clarke, G. \& Wright, G. (1964). Scheduling of vehicles from a central depot to number of delivery points. Operations Research. vol. 12, (1964), 568-581

[12]. Corella, J. Tactical automated security system (TASS): air force expeditionary security. Presented at the SPIE Conf. Unattended Ground Sensor Technologies and Applications (2003).

[13]. Cullen, F., Jarvis, J., and Ratliff, H. Set partitioning based heuristic for interactive routing. Networks, 11 (1981), $125-144$. 
[14]. Dhillon, S., Chakrabarty, K., and Iyengar, S. Sensor placement for grid coverage under imprecise detections. In Proceedings of the 5th International Conference on Information Fusion, vol. 2, (2002), 1581-1587.

[15]. Fisher, M., and Jaikumar, R. A generalized assignment heuristic for solving the VRP. Networks, 11 (1981), 109124

[16]. Foster, B., and Ryan, D. An integer programming approach to the vehicle scheduling problem. Operational Research Quarterly, 27, (1976), 367-384.

[17]. Gupta, P., and Kumar, P. The capacity of wireless networks. IEEE Transactions on Information Theory, 46, 2 (2000), 388-404.

[18]. Hu, W., Chou, C., Jha, S., and Bulusu, N. Deploying longlived and cost-effective hybrid sensor networks. In Proceedings of the First Workshop on Broadband Advanced Sensor Networks (2004), 749-767.

[19]. Isler, V., Daniilidis, K., and Kannan, S. Sampling based sensor-network deployment. In Proceedings of IEEE/RSJ International Conference on Intelligent Robots and Systems (2004), 1780-1785

[20]. Kornilakis, H. and Stamatopoulos, P. Crew pairing optimization with genetic algorithms. In Proceedings of the Second Hellenic Conference on AI: Methods and Applications of Artificial intelligence, 17 (2002), 375383.

[21]. Kumar, R., Tsiatsis, V., and Srivastava, M. Computation hierarchy for in-network processing. ACM WSNA, San Diego, CA, September (2003), 505- 518 .

[22]. Laporte, G., The vehicle routing problem: An overview of exact and approximate algorithms, European $J$. Operation. Research. 59 (1992), 345-358.

[23]. Lee, J., Krishnamachari, B., and Jay, K. Impact of heterogeneous deployment on lifetime sensing coverage in sensor networks. In Proceedings of IEEE SECON (2004), 367- 376.

[24]. Liu, X., and Mahapatra, P. On the deployment of wireless sensor nodes. In Proceedings of the Third International Workshop on Measurement, Modeling, and Performance Analysis of Wireless Sensor Networks, in conjunction with MobiQuitous, ACM Press, (2005).

[25]. Mainwaring, A., Polastre, J., Szewczyk, R., Culler, D., and Anderson, J. Wireless sensor networks for habitat monitoring. In Proceedings of the Intl. Workshop on Wireless Sensor Networks and Applications (2002), 88-97.

[26]. Mauri, K., Marko, H, and Timo, D. A Survey of application distribution in wireless sensor networks. EURASIP Journal on Wireless Communications and Networking, Special Issue on Ad Hoc Networks: CrossLayer Issues, issue 5, (2005), 774-788.

[27]. Mhatre V., and Rosenberg, C. Design guidelines for wireless sensor networks: communication, clustering and aggregation. Ad Hoc Networks Journal, Elsevier Science, 2 (2004), 45-63.

[28]. Mhatre, V., Rosenbert, C., Kofman, D., Mazumdar, R., and Shroff, N. A minimum cost heterogeneous sensor network with a lifetime constraint. IEEE Transactions on Mobile Computing,4 (2004), 4-15.
[29]. Nguyen, N., Venkatesh, S., West, G., and Bui, H. Multiple camera coordination in a surveillance system. Acta Automatic Sinica, 29, 3 (2003), 408-422.

[30]. O'Rourke, J. Galleries need fewer mobile guards: a variation on Chvatal's theorem. Geometriae Dedicata, 14 (1983), 273-283.

[31]. Ramadan, R., Abdelghany, K., El-Rewini, H. Optimal and approximate approaches for deployment of heterogeneous sensing devices. In EURASIP JWCN Journal, special issue in Mobile Multi-hop Ad hoc Networks, (2007), 3649.

[32]. Schurgers, C., Tsiatsis, V., Ganeriwal, S. and Srivastava, M. Topology management for sensor networks: exploiting latency and density. In Proceedings of ACM MobiHoc (2002), 135-145.

[33]. Subramanian, R. and Fekri, F. 2006. Sleep scheduling and lifetime maximization in sensor networks: fundamental limits and optimal solutions. In Proceedings of the Fifth international Conference on information Processing in Sensor Networks (2006), 218-225.

[34]. Toth, P. and Vigo, D., Eds. The Vehicle Routing Problem. Society for Industrial and Applied Mathematics, 2001.

[35]. Wang, G., G. Cao, and T. F. La Porta, Movement-assisted sensor deployment. IEEE Transactions on Mobile Computing, vol. 5, no. 6, (2006), 640-652.

[36]. Wang, G., Cao, G., and Porta, T. A Bidding Protocol for Deploying Mobile Sensors. In Proceedings of the 11th IEEE International Conference on Network Protocols (ICNP), 2003, 315-324.

[37]. Xu, K., Hong, X., and Gerla, M. An ad hoc network with mobile backbones. In Proceedings of IEEE International Conference on Communications (2002). 3138-3143.

[38]. Xu, Y., Heidemann, J., and Estrin, D. Geography-informed energy conservation for ad hoc routing. In Proceedings of MobiCom '01 (2001).

[39]. Yang, X., and Vaidya, N. A wakeup scheme for sensor networks: achieving balance between energy saving and end-to-end delay. In Proceedings of the 10th IEEE RealTime and Embedded Technology and Applications Symposium (2004), 19-26.

[40]. Ye, W., Heidemann, J., Estrin, D. An energy-efficient MAC protocol for wireless sensor networks. In Proceedings of IEEE INFOCOM, (2002), 1567-1576. 